\title{
Rhombencephalitis with Listeria monocytogenes
}

\author{
Aslıhan Yerlikaya², Baldan Eryılmaz², Bülent Kahyaoğlu³, Önder Ergönül4 \\ 1 Department of Hematology, The University of Texas MD Anderson Cancer Center, Texas, USA \\ 2 Department of Internal Medicine, Kocaeli University, Kocaeli, Turkey \\ 3 Department of Neurology, American Hospital, İstanbul, Turkey \\ ${ }^{4}$ Department of Infectious Diseases and Clinical Microbiology, Koç University School of Medicine, İstanbul, Turkey
}

\begin{abstract}
We described clinical course and outcome of a patient with Listeria monocytogenes meningitis and rhombencephalitis (RE). A 65-year-old woman with past medical history of chemotherapy and radiotherapy for cervical cancer applied to emergency room with the history of fever and headache for three days. Clinical course indicating meningitis evolved to RE over time. We present this case because of the significance of early diagnosis and adequate antimicrobial therapy targeted to the pathogen. At the end, we suggested an algorithm for the case management.

Keywords: Listeria monocytogenes, rhombencephalitis, immunocompromised host
\end{abstract}

\section{INTRODUCTION}

L isteriosis is a primarily foodborne but preventable disease caused by Listeria monocytogenes, a gram positive, facultative anaerobic and non-spore formIng bacillus that is ubiquitous in soil and water (1). L. monocytogenes was isolated and described in laboratory pigs and rabbits by Murray for the first time in 1929 (2). Especially since 1980s, L. monocytogenes has been recognized due to devastating widespread foodborne outbreaks and invasive life threatening diseases in immunocompromised patients (3). L. monocytogenes is mainly transmitted via contaminated food such as poultry, dairy products, fresh soft cheese, processed meat and raw vegetables $(4,5)$.

Majority of listeriosis cases occur in predisposed individuals including neonates, elderly, pregnant women and immunocompromised patients associated with malignancy, Acquired Immunodeficiency Syndrome (AIDS), glucocorticoid therapy, and transplantation history. Clinical presentations of listeriosis change with wide spectrum range from mild-influenza like illness to fatal sepsis (5). Systemic diseases including meningitis, rhombencephalitis (RE), endocarditis and bacteremia are primarily seen in these susceptible hosts whereas self-limited febrile gastroenteritis (GE) occurs in normal healthy individuals (4).

Listeriosis is acknowledged as the third fatal foodborne infection with 19\% mortality rate (6). Furthermore, L. monocytogenes meningitis is the third most common bacterial

\section{Corresponding Author: Önder Ergönül \\ E-mail: oergonul@ku.edu.tr}

Received: March 30, 2019 Accepted: May 23, 2019 Published: May 30, 2019

Suggested citation: Yerlikaya A, Eryılmaz B, Kahyaoğlu B, Ergönül Ö. Rhombencephalitis with Listeria monocytogenes. Infect Dis Clin Microbiol 2019; 1 : 47-54.

DOI: $10.5152 / \mathrm{idcm} .2019 .19008$ 
meningitis (5-10\%) with a mortality rate up to $50 \%$ and a neurologic sequel rate of $60 \%$ despite of the antibiotic treatment (5).

L. monocytogenes is known as the most common infectious causative agent of RE (7). The term RE refers to an inflammatory disease affecting the hindbrain (brainstem and cerebellum). The RE has a wide variety of etiologies with some of them potentially severe and life threatening without a proper early treatment (7). The overall mortality rate of L. monocytogenes RE is as high as $51 \%$, whereas all untreated cases end up with death. The success rate of $\mathrm{L}$. monocytogenes $\mathrm{RE}$ can reach up to $70 \%$ with appropriate early treatment (8). Therefore, considering possibility and starting empiric treatment for L. monocytogenes that is the most treatable etiologic factor for RE is highly fundamental. By this report, we present the case of invasive L. monocytogenes meningitis that latterly progressed to RE in immunocompromised patient. We updated clinical features, diagnosis and treatment options of this rare disease.

\section{CASE PRESENTATION}

A 65-year-old woman with inoperable cervical carcinoma was admitted to the Emergency Department of American Hospital, İstanbul, Turkey on July $20^{\text {th }} 2015$ with three days history of headache and fever. She recently received chemotherapy and radiotherapy. At the time of admission, there was no pathological neurological physical finding. The patient was hospitalized because of the patient's risk factors associated with her past medical history.

\section{HIGHLIGHTS}

- Early recognition of rhombencephalitis is important.

- Administration of adequate antimicrobial therapy is crucial in management of patients with central nervous system listeriosis.

- Close follow-up of patient's condition, repeating magnetic resonance imaging and managing complications are fundamental in case management.

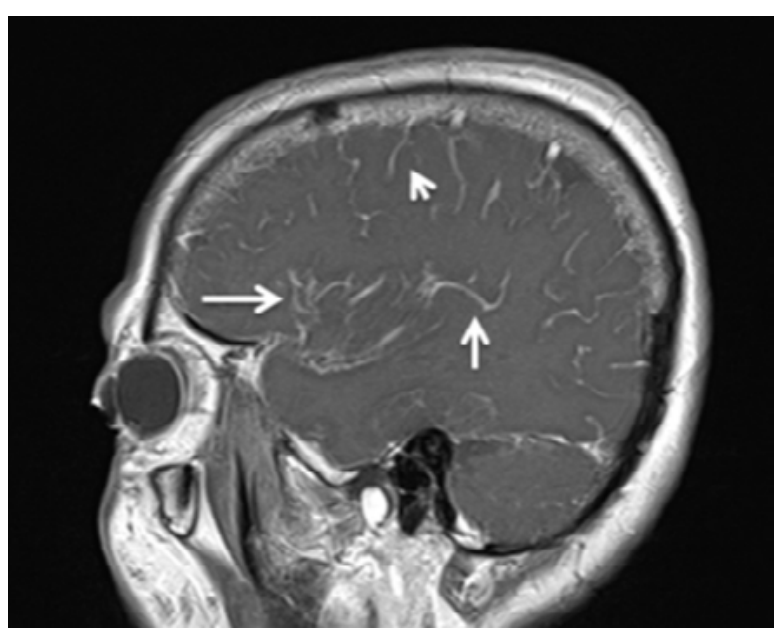

Figure 1. Sagittal plane T1 MRI image after contrast injection showed diffuse and increased enhancement of arachnoid matter (white arrows)

One day later, positive Brudzinski and Kernig signs and absence of verbal response were observed in patient's neurologic exam. Cranial Magnetic Resonance Imaging (MRI) demonstrated leptomeningeal enhancement which was indicating meningitis (Figure 1).

White blood cell (WBC) count was 11.85 x103/uL (3.8-10.8 x103/uL), hemoglobin (Hb); $12.1 \mathrm{~g} / \mathrm{dL}$ (12$15.5 \mathrm{~g} / \mathrm{dL}$ ), hematocrit; 35.1\% (36-47\%), Platelet count; $88 \times 10^{3} / \mathrm{uL}$ (130-400 x103/uL), calcium; 8.3 $\mathrm{mg} / \mathrm{dL}$ (8.5-10.3 mg/dL), creatinine; $0.4 \mathrm{mg} / \mathrm{dL}$ (0.6$1.1 \mathrm{mg} / \mathrm{dL})$, magnesium; $1.89 \mathrm{mEq} / \mathrm{L}$ (1.5-2.5 mEq/L), aPTT; 25sec (20-40 sec), international normalized rate (INR); 1.06 (0.9-1.2), C-reactive protein (CRP); $212.8 \mathrm{mg} / \mathrm{L}(<3 \mathrm{mg} / \mathrm{L})$.

Cerebrospinal fluid (CSF) examination supported the diagnosis for meningitis. In CSF, glucose level was $50 \mathrm{mg} / \mathrm{dL}$, simultaneous blood glucose was 100 $\mathrm{mg} / \mathrm{dL}$, protein: $239.3 \mathrm{mg} / \mathrm{dL}$ (15-45 mg/dL), erythrocytes; 2 (x103/uL), WBC; 3.92 (x103/uL), 86\% neutrophil, 13\% lymphocyte, $2 \%$ monocyte, and no acid resistant bacteria, no atypical cell was detected.

Based on blood and CSF test results, meningitis was considered as an initial diagnosis. Treatment with meropenem $6 \mathrm{~g}$ q $24 \mathrm{~h}$ was started and later vancomycin $1 \mathrm{~g}$ IV q $12 \mathrm{~h}$ was added. The patient's general condition had worsened with persistent fever de- 


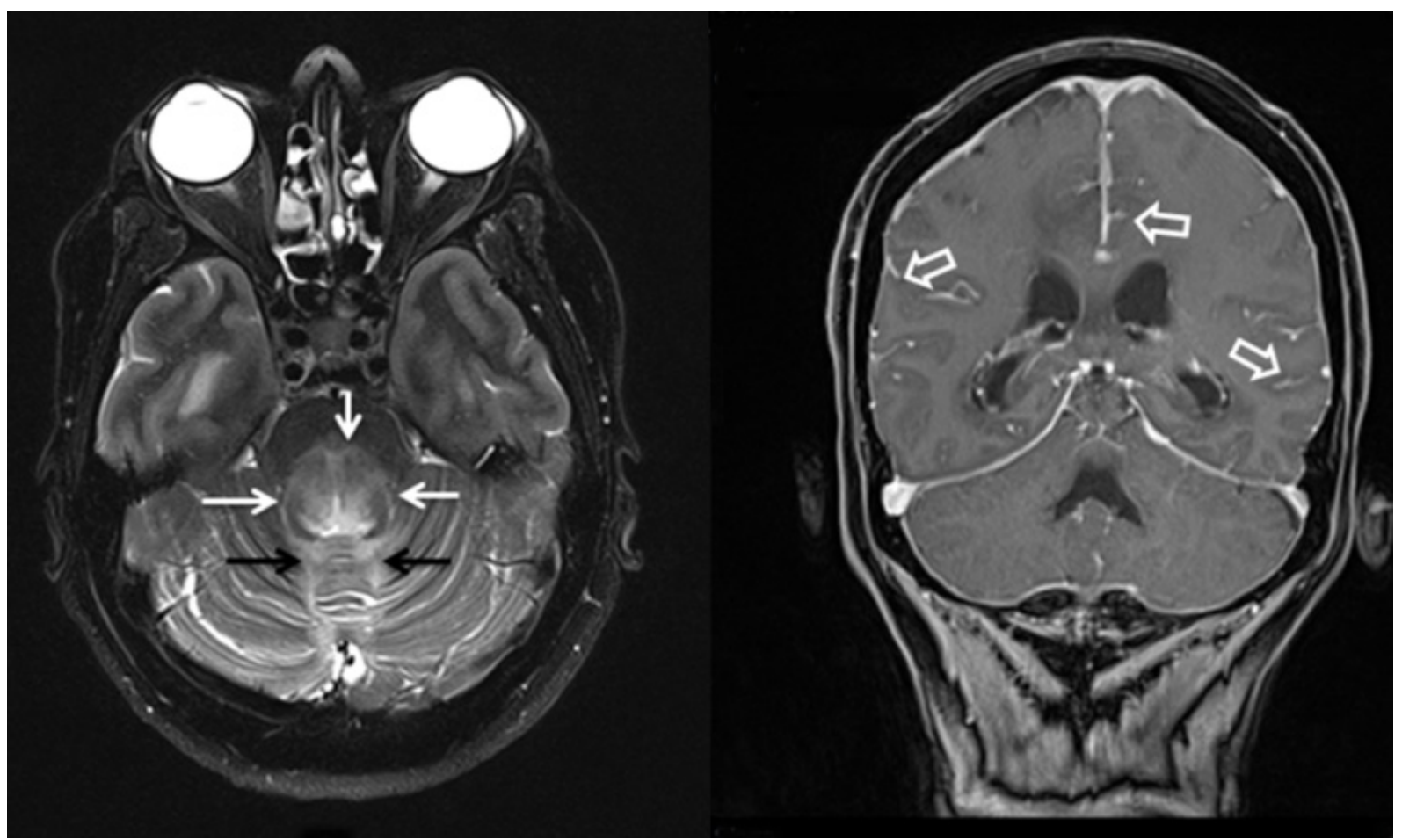

Figure 2. A) T2 weighted axial image showed hyperintensity of posterior pons (white arrows) and also cerebellar vermis (black arrows); B) Sagittal plane T1 weighted image demonstrated pial and arachnoid enhancement (open arrows)

spite of the therapy. Repeated cranial MRI revealed advanced leptomeningeal enhancement compared with the previous one. In neurologic exam, muscle strength was 3 out of 5 . Bilateral positive Babinski sign (bilateral extension) and nuchal rigidity were present in physical examination. Pronator drift (Barre's sign) and lower extremity weakness were prominent. Ophtalmoscopic exam revealed deficits in lateral eye movements and diplopia in left direction. Blurring of optic margins indicating papilledema was detected. The patient was monitorized and treated in Intensive Care Unit (ICU) due to severity of her clinical course and prominent neurological signs.

In CSF culture, L. monocytogenes was isolated. Ampicillin $2 \mathrm{~g} \mathrm{q} 6 \mathrm{~h}$ plus gentamicin $80 \mathrm{mg}$ q $8 \mathrm{~h}$ were started. Despite of the antibiotic treatment, patient's clinical condition had been worsening with Glascow coma score (GCS) of 10 and Acute Physiology and Chronic Health Evaluation (APACHE-II) score of 16. Prominent neurologic exam findings were muscle strength of $1 / 5$, hypotonia, presence of pathological reflexes, positive bilateral Babinski sign, anisocoria, $6^{\text {th }}$ nerve abducens paralysis, nystagmus and absence of extraocular movements. This clinical condition indicated us increased intracranial pressure and hydrocephalus. Repeated MRI findings demonstrated hydrocephalus and increased intracranial edema. External ventricular drain (EVD) was placed to relieve intracranial pressure (ICP) on $27^{\text {th }}$ of July 2015 and intracranial pressure levels turned to normal values (5-15 mmHg). Meantime, levetirecetam was given due to patient's seizures. The patient was intubated and ventilated because of her impaired neurologic and respiratory function. ICP levels returned to normal range with EVD but repeated MRI results revealed progression of RE spreading towards to medulla spinalis.

Patient's treatment of ampicillin and gentamicin combination was continued under close monitorization. However, her clinical condition had worsened with 0-1/5 muscle strength, quadriparesis, oculomotor and abducens nerve palsies, GCS of 3 and APACHE-II of 21 scores. Because of severe neurological deficits and brain edema, mannitol treatment was started. Rifampicin 600 mg q 24h was added to 
antibiotic regimen on the $10^{\text {th }}$ day of antibiotic therapy to reduce risk for treatment failure. The patient was given ampicillin $2 \mathrm{~g} \mathrm{q} 6 \mathrm{~h}$ and gentamicin $80 \mathrm{mg}$ q 8 h for 6 weeks.

\section{DISCUSSION}

\section{Epidemiology}

Listeriosis is a public health problem due to epidemic foodborne outbreaks and high mortality rate up to $30 \%$. The risk factors for listeriosis are extremes of age ( $<2$ or $>60$ ), pregnancy, hematologic and solid malignancies, chemotherapy agents like fludarabine, corticosteroid treatment, diabetes, chronic kidney or liver disease also other chronic diseases (collagen vascular disease etc.), AIDS, transplantation history and immunosuppressive treatment (anti-Tumor Necrosis Factor- $\alpha$ agents etc.) history (1).

Epidemiological studies indicate an increasing trend in incidence rates predominantly in Europe. Inadequate pasteurization technique, centralized food processing causing widespread consumption of contaminated products especially ready to eat meat or dairy products and reduced salt content in food have been attributed to this increase (4). According to 2013 Centers for Disease Control and Prevention (CDC) reports, incidence of listeriosis cases in USA was 0.26 per 100.000 individuals with total 1600 cases and 260 deaths (9).

L. monocytogenes species is ubiquitously found in soil, water, decayed substances and $5 \%$ in human gastrointestinal flora (4). Approximately 99\% of listeriosis cases are contaminated trough consumption of contaminated food markedly fresh soft cheese, processed meat/seafood products, apple cider, coleslaw, raw vegetables and less commonly through direct contact with diseased animals and their products (1). Ingestion of pathogen leads to gastrointestinal mucosa invasion and systemic spread in predisposed individuals.

In our case, patient had multiple risk factors for immunosupression including age, solid cervical carcinoma and immunosuppressive treatment history, absence of trimethoprim/sulfamethoxazole (TMP/SMX) prophylaxis and use of proton pump inhibitor (PPI) similar to previous studies (10). There was pertinent history of soft cheese consumption which was accepted as high-risk food for listeriosis.

\section{Microbiology}

L. monocytogenes is the only one among Listeria species that causes to recurrent infections in humans. L. monocytogenes is rod-shaped, Gram positive, intracellular, facultative anaerobic bacillus. It is motile by its flagella at $15-30{ }^{\circ} \mathrm{C}$ but not at $37^{\circ} \mathrm{C}$. It has properties of catalase positive and oxidase negative. When grown on sheep blood agar, it is weakly $\beta$ - hemolytic owing to presence of $\beta$-hemolysin (4). L. monocytogenes can replicate in wide spectrum of temperature ranging with 1 to $45^{\circ} \mathrm{C}$ and under high salt concentrations and refrigerator conditions leading to devastating food borne outbreaks (4).

Table 1. Clinical parameters on the day of hospital admission and during follow-up

\begin{tabular}{|l|c|c|c|c|c|}
\hline Parameters & $\begin{array}{c}\text { Hospital } \\
\text { admission } \\
\text { (Day 0) }^{\text {a }}\end{array}$ & Day 3 & Day 6 & Day 10 & Day 40 \\
\hline $\begin{array}{l}\text { Body } \\
\text { Temperature } \\
\left({ }^{\circ} \mathrm{C}\right)\end{array}$ & 39 & 38 & 38.3 & 36.7 & 37.2 \\
\hline $\begin{array}{l}\text { C-reactive } \\
\text { protein } \\
\text { (mg/L) }\end{array}$ & 357 & 456 & 170 & 220.6 & 28.9 \\
\hline $\begin{array}{l}\text { Leukocyte } \\
\text { count } \\
\left(\mathrm{x} 10^{3} / \mathrm{uL}\right)\end{array}$ & 11.85 & 11 & 5.5 & - & 11.32 \\
\hline $\begin{array}{l}\text { APACHE-II } \\
\text { score }\end{array}$ & 22 & 22 & 16 & 14 & - \\
\hline $\begin{array}{l}\text { Glasgow } \\
\text { coma scale }\end{array}$ & 13 & 13 & 10 & 6 & 12 \\
\hline
\end{tabular}

a Patient was hospitalized due to meningitis suspicion. Meropenem2g q $8 \mathrm{~h}+$ Vancomycin $1 \mathrm{~g}$ IV q $12 \mathrm{~h}$ was started

${ }^{b}$ CSF/Blood culture incubated L. monocytogenes; ampicillin $2 \mathrm{~g} q 6 \mathrm{~h}+\mathrm{gentamicin} 80 \mathrm{mg}$ q 8 h was started

${ }^{c}$ Admitted to ICU due to worsened clinics. External ventricular drainage was placed due to increased ICP.

${ }^{d}$ Rifampicin $600 \mathrm{mg}$ q $24 \mathrm{~h}$ was added to treatment regime

e Stable neurological condition, 6th week of ampicillin + gentamicin treatment

Normal range for $\mathrm{CRP}<3 \mathrm{mg} / \mathrm{L}$; for WBC: $3.8-10.8 \times 10^{3} / \mathrm{LL}$

APACHE: score of $>25$ represents predicted mortality of $50 \%$, score of $>35$ represent predicted mortality of $80 \%$ 


\section{Pathogenesis}

L. monocytogenes has several different virulence mechanisms including intracellular growth, endotoxin production and presence of hemolysin or catalase enzymes $(1,11)$. After entry to host, L. monocytogenes invade primarily intestinal mucosa cells via binding to E-cadherin with their surface protein called internalin. After penetrating to host cell, it migrates to cytoplasm and vacuole $(1,11)$. Type of hemolysin called Listeriolysin $\mathrm{O}$ (endotoxin) is the main virulence factor providing ability of reducing cell $\mathrm{pH}$, causing pores and disrupting host cell membrane. Endotoxin composed of cholesterol and lipopolysaccharide impedes host immune response and induces $\mathrm{T}$ cell immunity, neutrophils and macrophages $(1,5)$. Owing to actin and polymerization mechanism, pathogen is able to spread by cell to cell without exposing to external environment and host's immune system (1).

\section{Clinical features}

Incubation period ranges from 18-20 hours (for gastroenteritis) to 1-4 weeks (for invasive diseases), which makes diagnosis difficult (4). Clinical presentation of L. monocytogenes has wide spectrum ranging from influenza-like mild illness, mild gastroenteritis to rhombencephalitis, meningitis and sepsis to death. For this reason, it is also described as "a disease of bimodal severity". In normal healthy population, L. monocytogenes is attributed to $1 \%$ of febrile gastroenteritis cases with symptoms of watery diarrhea, fever, nausea, headache, myalgias and arthralgias. It is usually self-limited with 2-day course and resolves with complete recovery (4).

Life-threatening CNS infections including meningitis, meningoencephalitis, and rhombencephalitis are observed among predisposed population. L. monocytogenes meningitis is accepted as the third most common cause of meningitis. Main clinical features are headache, fever, nuchal rigidity, seizures, tremor, ataxia and altered mental status. During its course, symptoms can progress to cranial nerve palsies, hemiparesis, coma and death most resulting from a complication of increased intracranial pressure (12).

The RE has detrimental consequences with mortality rate of $51 \%$ and significant neurologic se- quel of $60 \%$ among survivors (8). Clinical course usually starts with prodromal non-specific period such as fever, headache, neck stiffness, nausea or vomiting etc. Then clinical course progresses with severe neurological deficits ranging from cranial nerve palsies (particularly $3^{\text {rd }}, 6^{\text {th }}$ and $7^{\text {th }}$ nerves), pathological reflexes to more severe involvement of spinal cord tracts leading to hemi or quadriparesis, sensory deficit paresthesia, respiratory failure, dysphagia, dysarthria and ataxia $(8,13,14)$.

Our patient's clinical condition followed two phases. Fever, headache, and positive Brudzinski and Kernig sign indicating nuchal rigidity were only prominent findings in the first phase similar to other bacterial meningitis cases. In the second phase of disease, hydrocephalus, seizures, unconsciousness, deficits of light/verbal response, cranial nerve palsies, abnormal extraocular movements, impaired muscle strength, pathological reflexes, positive Babinski sign and respiratory failure were noted directing us to consider rhombencephalitis.

\section{Diagnosis}

L. monocytogenes is diagnosed by a cerebrospinal fluid or blood culture of organism that is analyzed based on morphologic, motility and hemolytic properties of pathogen. It is widely accepted as a gold standard (15). Positive CSF examination reveals pleocytosis, elevated protein levels and low glucose levels (4). At the same time, there can be no or mild abnormalities in CSF findings in L. monocytogenes RE making diagnosis more difficult. Therefore, CSF findings and cultures cannot be used as exclusion criteria for L. monocytogenes RE (14). In our case, the blood and CSF analyses of the patient were similar to previous listeriosis reports (10). It is important to emphasize that antibodies based Enzyme Linked Immunosorbent Assay (ELISA) test and molecular Polymerase Chain Reaction (PCR) test provide more rapid results with equal sensitivity.

From radiological perspective, MRI with contrast is preferred for patients with suspicion of L. monocytogenes CNS infection. Cranial imaging demonstrates leptomeningeal and parenchymal (cerebellum, brainstem etc.) enhancement on T2 intensity window. Contrast enhanced T1 MRI can 
Figure 3. Recommended algorithm for rhombencephalitis

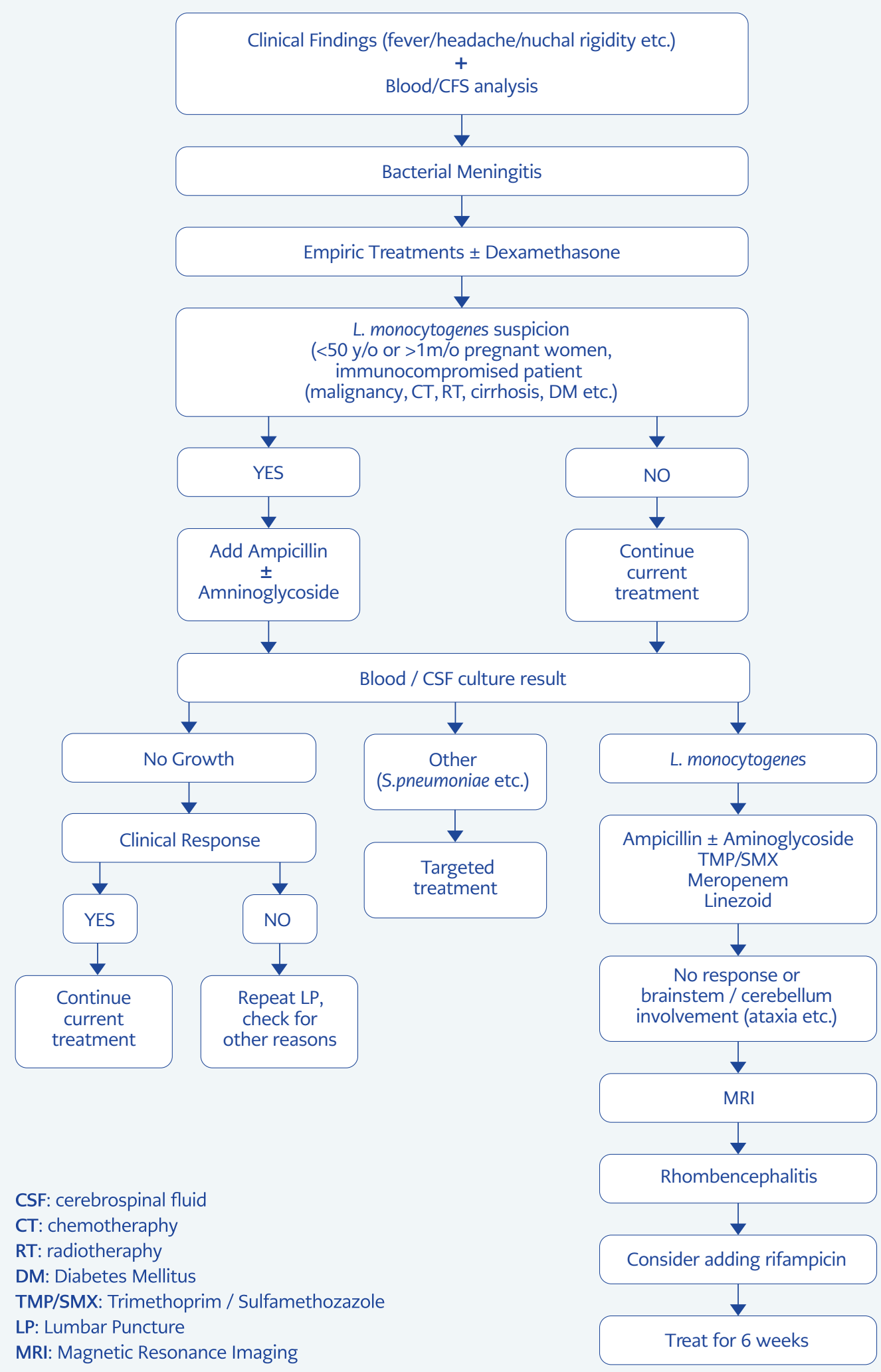


show cranial nerve enhancement, ring enhancement leading to abscess or heterogeneous hindbrain involvement (10).

Other reported causes of RE are listed as multiple sclerosis, Behçet disease, paraneoplastic syndrome, Epstein-Barr virus, tuberculosis, pneumococcal infection, Systemic Lupus Erythematosus, lymphoma, Brucella, JC virus and relapsing polychondritis (7).

\section{Therapy}

From treatment perspective, empirical vancomycin combined with cefotaxime or ceftriaxone therapy should be started on the basis of classic clinical presentation of CNS infection, MRI findings of leptomeningeal enhancement, later CSF blood analysis findings (pleocytosis, high protein content) and Gram positive CSF staining (10, 12). Furthermore, under presence of risk factors addition of ampicillin with or without aminoglycoside combination should be considered. Ampicillin addition is essential since vancomycin and cephalosporin have no or low activity against $\mathrm{L}$. monocytogenes. For patients unable to take $\beta$-lactam, TMP/SMX should be preferred.

In the management of L. monocytogenes CNS infections, treatment choice is generally ampicillin in combination with aminoglycosides preferably gentamicin. Other second-line alternative drugs are TMP/SMX, fluoroquinolones and meropenem (16). Thonnings et al. (17) revealed that ampicillin and gentamicin combination is more effective and has lower mortality rate compared with meropenem treatment. Further, rifampin has considerable bacteriostatic activity against L. monocytogenes and more penetrance to blood brain barrier compared with aminoglycosides. To prevent emergence of resistance, use of rifampin combination with ampicillin should be considered. Study results support to continue at least 3 weeks for L. monocytogenes meningitis and longer nearly 4-6 weeks for more invasive CNS like RE and abscess (16). We summarized our recommendations in Figure 3.
Another fundamental aspect to discuss for L. monocytogenes CNS infection is the development of obstructive hydrocephalus leading to seizures as observed in our case. When blood sodium level was analyzed, hyponatremia was observed. Hyponatremia can be associated with inappropriate Antidiuretic Hormone secretion usually seen in CNS infection or edema cases. Increased ICP can be a probable cause of higher neurological sequel and mortality rate in L. monocytogenes CNS infections. Meticulous observation of blood sodium levels and signs of increased ICP is extremely crucial.

Although, some of previous studies pointed out an advantage of corticosteroid (dexamethasone) administration as a prophylaxis before starting antibiotic treatment, there is a discordance and no sufficient evidence for L. monocytogenes meningitis cases (16). Presently, prophylactic dexamethasone has been recommended only for suspected $\mathrm{S}$. pneumoniae meningitis cases. In addition, since most CNS listeriosis occurs among immunocompromised patients, use of additional immunosuppressive steroid therapy should be investigated more deeply.

Seizures may also lead to increased mortality and morbidity rates. Therefore, prophylactic anticonvulsant use also should be studied in more detail (18).

In conclusion, early recognition of clinical findings of CNS infections, performing rapid diagnostic methods, and an administration of adequate antimicrobial therapy can be defined as cornerstones in management of patients with CNS listeriosis. Close follow-up of patient's condition, repeating MRI in the case of no improvement or clinical deterioration and managing complications are fundamental. Due to possible brainstem involvement leading to respiratory failure risk, patient's respiratory function should be closely followed up and adequate ventilation should be provided to patients. 
Informed Consent: Written informed consent was obtained from the patient who participated in this study.

Peer-review: Externally peer-reviewed.

Author Contributions: Concept - A.Y., B.E., B.K., Ö.E.; Design - A.Y., B.E., B.K., Ö.E.; Supervision - A.Y., B.E., B.K., Ö.E.; Materials - B.E., B.K.; Data Collection and/or Processing - B.E., B.K.; Analysis and/or
Interpretation - A.Y., B.E., B.K., Ö.E.; Literature Search - A.Y.; Writing Manuscript - A.Y., Ö.E.; Critical Review - A.Y., Ö.E.

Conflict of Interest: The authors have no conflict of interest to declare.

Financial Disclosure: The authors declared that this study has received no financial support.

\section{REFERENCES}

1 Farber JM, Peterkin PI. Listeria monocytogenes, a food-borne pathogen. Microbiol Rev 1991; 55: 476-511.

2 Hernandez-Milian A, Payeras-Cifre A. What is new in listeriosis? BioMed Res Int 2014; 2014: 358051-358051.

3 Ruggieri S, Logoteta A, Martini G, Bozzao A, De Giglio L. Listeria monocytogenes-Induced Rhombencephalitis in a Patient With Multiple Sclerosis Treated With Dimethyl Fumarate. JAMA Neurol 2018; 75: 762-3.

4 Wing EJ, Gregory SH. Listeria monocytogenes: clinical and experimental update. J Infect Dis 2002; 185(Suppl 1): 18-24.

5 Cartwright EJ, Jackson KA, Johnson SD, Graves LM, Silk BJ, Mahon BE. Listeriosis outbreaks and associated food vehicles United States, 1998-2008. Emerg Infect Dis 2013; 19: 1-9; quiz 184.

6 Bennion JR, Sorvillo F, Wise ME, Krishna S, Mascola L. Decreasing listeriosis mortality in the United States, 1990-2005. Clin Infect Dis 2008; 47: 867-74.

7 Moragas M, Martínez-Yélamos S, Majós C, Fernández-Viladrich P, Rubio F, Arbizu T. Rhombencephalitis: a series of 97 patients. Medicine (Baltimore) 2011; 90: 256-61.

8 Armstrong RW, Fung PC. Brainstem encephalitis (rhombencephalitis) due to Listeria monocytogenes: case report and review. Clin Infect Dis 1993; 16: 689-702.

9 Centers for Disease Control and Prevention. Incidence and trends of infection with pathogens transmitted commonly through food - foodborne diseases active surveillance network, 10 U.S. sites, 1996-2012. Morb Mortal Wkly Rep 2013; 62: 283-7.

10 Brouwer MC, van de Beek D, Heckenberg SG, Spanjaard L, de
Gans J. Community-acquired Listeria monocytogenes meningitis in adults. Clin Infect Dis 2006; 43: 1233-8.

11 Drevets DA, Sawyer RT, Potter TA, Campbell PA. Listeria monocytogenes infects human endothelial cells by two distinct mechanisms. Infect Immun 1995; 63: 4268-76.

12 van de Beek D, de Gans J, Spanjaard L, Weisfelt M, Reitsma JB, Vermeulen M. Clinical features and prognostic factors in adults with bacterial meningitis. N Engl J Med 2004; 351: 184959.

13 Jubelt B, Mihai C, Li TM, Veerapaneni P. Rhombencephalitis / brainstem encephalitis. Curr Neurol Neurosci Rep 2011; 11 : 543-52.

14 Workman S, Theal M. Rhomboencephalitis caused by Listeria monocytogenes. Can J Infect Dis 1997; 8: 113-6.

15 Gasanov U, Hughes D, Hansbro PM. Methods for the isolation and identification of Listeria spp. and Listeria monocytogenes: a review. FEMS Microbiol Rev 2005; 29: 851-75.

16 Tunkel AR, Hartman B, Kaplan SL, Kaufman BA, Roos KL, Scheld WM, et al. Practice guidelines for the management of bacterial meningitis. Clin Infect Dis 2004; 39: 1267-84.

17 Thønnings S, Knudsen JD, Schønheyder HC, Søgaard M, Arpi M, Gradel KO, et al. Antibiotic treatment and mortality in patients with Listeria monocytogenes meningitis or bacteraemia. Clin Microbiol Infect 2016; 22: 725-30.

18 Amaya-Villar R, García-Cabrera E, Sulleiro-Igual E, Fernández-Viladrich P, Fontanals-Aymerich D, Catalán-Alonso P, et al. Three-year multicenter surveillance of community-acquired Listeria monocytogenes meningitis in adults. BMC Infect Dis 2010; 10: 324 